\title{
Formación on line
}

\author{
O. Grau-Perejoan
}

\begin{abstract}
La formación on line es una modalidad de enseñanza a distancia basada en las nuevas tecnologías. En este artículo se pretende hacer una introducción a base de describir a grandes rasgos sus características principales: asincronía, no presencialidad, comunicación escrita, función del profesor on line, así como los retos, los riesgos, las ventajas y los inconvenientes que plantea. Se exponen las diferencias entre la formación on line y la formación presencial, de manera que los docentes puedan adaptar de la mejor manera posible sus propuestas formativas a la modalidad on line. Se introduce el importantísimo papel de la planificación y de la fase de diseño y, finalmente, se repasan conceptos útiles para comprender mejor el mundo de la formación on line como son los conceptos entorno virtual de aprendizaje (EVA) o Blended Learning (B-Learning).
\end{abstract}

Palabras clave. E-learning. Formación on line. Formación presencial.

\section{On line learning}

On line learning is a type of distance education based on new technologies. This article's aim is to introduce its main characteristics - asynchrony, non-presentiality, written communication, e-teacher role-as well as its challenges, risks, advantages and limitations. Differences between on line learning and face-to-face learning are presented in order to enable educational professionals to adapt their courses to the on line methodology. Planning and designing are introduced as key phases and, finally, useful concepts such as Virtual Learning Environment (VLE) or Blended Learning (B-Learning) are reviewed in order to achieve a better understanding of the on line learning field.

Key words. E-learning. Face-to-face learning. On line learning.

\section{Introducción}

La expresión 'formación on line’ es quizás la fórmula más extendida para referirnos a soluciones de formación a distancia que usan Internet como canal de comunicación principal. Se trata de una modalidad de formación que contempla habitualmente un uso extensivo de las tecnologías de la información y la comunicación (TIC), que explota los diferentes recursos tecnológicos ofrecidos por Internet y que tiene como principales características distintivas el hecho de que se desarrolla en un espacio virtual y que la comunicación entre los participantes de las actividades se realiza a través de este espacio y/o a través de $e$-mail.

Existen muchas otras expresiones usadas como sinónimos o cuasi-sinónimos con diferentes matices como son formación virtual, web-based learning, computer-based learning, teleformación o e-learning, esta última la más popular entre ellas. Este artículo, sin embargo, se ciñe al tema de la formación a través de Internet, de ahí que se use la expresión formación on line, porque se ha considerado que el medio (Internet) es el elemento que ha hecho que este tipo de formación sea la modalidad de educación a distancia predominante.

\section{Principales características de la formación on line}

Del mismo modo que existen expresiones diferentes que, en el ámbito de la formación on line, se usan para hacer referencia a más o menos lo mismo, también existen definiciones diversas
Cuestionario

de acreditación

www.educmed.net
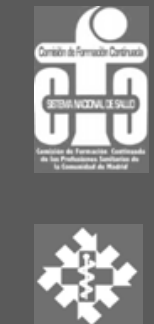

Jefe de la Unidad

de Formación on line.

Fundació Doctor

Robert-Universitat

Autònoma de

Barcelona. Barcelona

España.

Correspondencia

Oriol Grau Perejoan.

Fundació Doctor

Robert-UAB. St. Antoni

Maria Claret, 171

E-08041 Barcelona.

E-mail

oriol.grau@uab.es 
que no tienen porque coincidir con la que se acaba de proponer, pero que casi siempre contemplan las siguientes características como definitorias de esta modalidad de formación:

- No presencialidad.

- Asincronía.

- Uso extensivo de las TIC y de Internet.

Como buena heredera de la formación a distancia, el éxito que está obteniendo la formación on line -por lo menos a juzgar por las cifras que publican las grandes consultoras del sector $[1,2]-$ radica sobre todo en el hecho de que separa a los participantes de una actividad en el espacio y en el tiempo. Es decir, es un tipo de formación que es básicamente no presencial y asíncrona, aunque pueda tener diferentes cuotas de sincronía (chats) y presencialidad (encuentros a principio o final de curso). Estas dos cualidades, no presencialidad y asincronía, permiten romper las barreras de espacio y tiempo, respectivamente: los participantes no deben encontrarse a una determinada hora en un determinado sitio para garantizar su correcto desarrollo y, por tanto, el aprendizaje puede tener lugar en cualquier sitio a cualquier hora. Estas características, combinadas con el factor Internet, recurso tecnológico al alcance de la mayor parte de la población en nuestro país, explicarían en gran medida la gran aceptación que tiene la formación on line.

Estos rasgos distintivos dotan a la formación on line de unas particularidades en comparación con la formación presencial y, por tanto, de unas ventajas y límitaciones en relación con esta última:

\section{Ventajas}

- Se puede distribuir una actividad de formación a cualquier rincón del planeta.

- Ahorra costes en desplazamientos.

- Se puede hacer llegar una actividad de formación a segmentos de alumnos que tienen vetada la formación presencial por motivos geográficos o relacionados con los horarios.

- El alumno goza de mayor autonomía, decide cuándo y dónde va a ponerse a trabajar.

- Puede permitir que los estudiantes marquen su propio ritmo de aprendizaje.

- Tiende a ser más flexible que la formación presencial, tanto desde el punto de vista del alumno como del profesor.
- Facilita la actualización de la información y de los contenidos.

- Toda la actividad realizada por los estudiantes queda registrada en los servidores.

\section{Limitaciones}

- Requiere un gran esfuerzo y una importante inversión de recursos económicos y humanos en la fase de planificación.

- Implica diferentes roles profesionales que van más allá de los docentes: diseñadores, informáticos, dibujantes, pedagogos, además de profesores/tutores, directores, coordinadores y autores de material (expertos).

- Dificultades en la motivación de los estudiantes: los alumnos trabajan en soledad; la formación on line exige autodisciplina y puede haber sensación de aislamiento por parte del alumno.

- Los alumnos, acostumbrados al modelo tradicional de formación, necesitarán desarrollar nuevas estrategias de aprendizaje.

- En general, requiere más trabajo que la formación presencial, tanto para el profesor, que tendrá que dedicarse a sus alumnos diariamente mientras la actividad de formación tenga lugar, como para los alumnos.

- Requiere unas competencias tecnológicas mínimas por parte de los participantes, especialmente de los docentes.

- Desde el punto de vista relacional-social, la formación on line es claramente inferior a la formación tradicional porque las herramientas electrónicas de comunicación, aunque muy desarrolladas, no se pueden comparar todavía con la expresividad y los complejos matices de los recursos 'presenciales' de comunicación que tenemos los seres humanos.

- Puede haber sobrecarga de información.

- Dependencia tecnológica.

Para más información sobre ventajas, inconvenientes y comparación con la formación presencial, véase el artículo de Cabero [3].

\section{Comunicación}

Otra característica muy importante, pero que no se suele citar mucho, es que la comunicación en 


\title{
Programa de Formación Médica Acreditada en Educación Médica
}

\author{
Artículo: Formación on line
}

Autor: O. Grau-Perejoan

Revista: Educ Med 2008; 11 (3): 137-144

1. Indique la opción correcta. Según el autor, las características definitorias de la formación on line son:

$\square$ a) usar un entorno virtual de aprendizaje (EVA) y la no presencialidad

$\square$ b) asincronía, no presencialidad, uso extensivo de las tecnologías de la información y la comunicación (TIC) y de Internet y enfoque pedagógico

$\square$ c) asincronía, no presencialidad y uso extensivo de las TIC y de Internet

$\square$ d) enfoque pedagógico y uso extensivo de las TIC y de Internet

$\square$ e) usar un EVA, asincronía y no presencialidad

\section{De entre las opciones siguientes, una de ellas no ha sido} considerada una ventaja de la formación on line:

$\square$ a) el alumno goza de mayor autonomía

$\square$ b) permite hacer llegar una actividad de formación a cualquier rincón del planeta

$\square$ c) ahorra costes en desplazamientos

$\square$ d) en general requiere más trabajo que la formación presencial

$\square$ e) tiende a ser más flexible

\section{Respecto a la comunicación escrita}

¿cuál de las siguientes afirmaciones es falsa?

$\square$ a) tiene un potencial de reflexión alto

$\square$ b) es más informal que la comunicación oral

$\square$ c) tiene menos potencial para comunicar sensaciones y emociones

$\square$ d) perdura porque queda registrada en los servidores

$\square$ e) se pierde inmediatez respecto a la comunicación oral

4. Respecto a la planificación y al diseño de una actividad de formación on line, indicar la proposición falsa:

$\square$ a) implican a equipos pluridisciplinares de profesionales

$\square$ b) implican una inversión de recursos considerable

$\square$ c) es importante que tanto la planificación como el diseño hayan concluido en su totalidad antes del inicio de la actividad

$\square$ d) el profesor necesita que estas fases estén concluidas para poder planificar su trabajo

$\square$ e) la creación de los materiales didácticos no se incluyen en estas fases

5. Indique la opción correcta. Para llevar a cabo una actividad de formación on line necesitaremos contar con equipos de profesionales diferentes para:

$\square$ a) diseñar, impartir, administrar y coordinar

$\square$ b) diseñar, motivar, administrar y coordinar

$\square$ c) diseñar, guiar, facilitar y motivar

$\square$ d) diseñar, planificar, impartir y gestionar

$\square$ e) diseñar, impartir, controlar y administrar
6. Indique la opción correcta. Las funciones del profesor on line son:

$\square$ a) coordinadora, social, gestora y tecnológica

$\square$ b) tecnológica, motivadora, planificadora y pedagógica

$\square$ c) gestora, pedagógica, tecnológica y social

$\square$ d) pedagógica, motivadora, gestora y tecnológica

$\square$ e) motivadora, facilitadora, pedagógica y tecnológica

7. Indique la opción correcta. En función del acceso al código fuente existen dos grandes bloques de plataformas de aprendizaje o EVA; estos dos bloques son:

$\square$ a) software libre y comerciales

$\square$ b) comerciales y open source

$\square$ c) gratuitas y no gratuitas

$\square$ d) open source y propietarias

$\square$ e) comerciales y propietarias

8. El Blended Learning o B-learning es una combinación de formación presencial y on line; su punto fuerte reside en que:

$\square$ a) tiene mucho poder motivador porque a todo el mundo le gusta mezclar innovaciones tecnológicas y educación

$\square$ b) combina los bajos costes de la formación on line con el alto poder motivador de la formación presencial

$\square$ c) combina el alto poder motivador de la tecnología con la flexibilidad de la formación presencial

$\square$ d) es más divertido que la formación presencial

$\square$ e) combina la comodidad y flexibilidad de la formación on line con el alto poder motivador de la formación presencial

9. La combinación de la web 2.0 y formación on line consiste en:

$\square$ a) aplicar las últimas innovaciones tecnológicas a la formación on line

$\square$ b) hacer actividades colaborativas, como por ejemplo debates, para conseguir hacer de nuestro grupo de alumnos una verdadera comunidad de usuarios

$\square$ c) motivar a los alumnos para que participen y se relacionan y así conseguir crear un buen ambiente en el aula

$\square$ d) aplicar tecnologías de marcado carácter participativo y social, como por ejemplo blogs y wikis, a actividades de formación on line

$\square$ e) usar blogs para comunicarnos con nuestros alumnos en vez de las herramientas que nos proporcione nuestro EVA

10. Existen varios enfoques que conviene evitar cuando diseñemos una propuesta de formación on line. Uno de ellos consiste en:

$\square$ a) planificar nuestra actividad de formación

$\square$ b) realizar encuentros presenciales

$\square$ c) situar la tecnología por encima de las personas

$\square$ d) no realizar encuentros presenciales

$\square$ e) situar a las personas por encima de la tecnología

Nombre y apellidos

DNI . . .

Dirección

Código postal . .

Localidad....

Provincia/país

Teléfono

E-mail

Encontrará este cuestionario en la web de la revista: www.educmed.net 
formación on line es básicamente escrita. Éste es un elemento muy relevante porque tiene unas consecuencias pedagógicas y técnicas que se deben tener en cuenta y que modifican de forma sustancial la manera en que se desarrolla la actividad de formación:

- Perdurabilidad: todo queda registrado (muy útil para realizar las evaluaciones).

- Alto potencial de reflexión: al ser la comunicación escrita más planificada, más elaborada que la oral, tiende a incitar a la reflexión.

- Se pierde inmediatez, la interacción es más lenta.

- Más formal que la comunicación oral.

- Códigos no verbales: se pierden matices faciales, gestuales, de entonación, etc. que se pueden intentar sustituir por colores y diferentes recursos tipográficos como la disposición del texto, negritas, etc.

- Se pierde potencial para comunicar sensaciones y emociones.

- Los tímidos, que acostumbran a autoexcluirse en actividades presenciales, tienen en este tipo de comunicación menos impedimentos para participar y comunicarse.

Es importante tener en cuenta los puntos fuertes y débiles de la comunicación escrita para poder diseñar actividades de aprendizaje que se adapten correctamente a la formación on line. Algunos consejos para profesores en la comunicación con sus alumnos se presentan en la tabla I.

\section{Planificación}

Desde el punto de la vista del diseño, es importante ser consciente que en la formación on line la fase de preparación de un curso cobra todavía más importancia que la que pueda tener en un curso presencial. En una propuesta on line la fase de planificación y de diseño es especialmente compleja porque implica a equipos pluridisciplinares, que incluyen profesionales como diseñadores y técnicos informáticos. Estos perfiles son necesarios para dar forma a los materiales didácticos de acuerdo con el enfoque pedagógico escogido, para diseñar el curso en cuanto a estructura y para secuenciar y temporalizar los recursos didácticos (diseño instruccional). Por otro lado, el profesional que estará en contacto
Tabla I. Algunos consejos para profesores en la comunicación con sus alumnos.

\footnotetext{
- Dar respuesta lo más rápido posible, preferentemente en 24 horas como mucho

- Utilizar un lenguaje conciso con frases preferentemente cortas

- Redactar mensajes estructurados

- Enfocar los temas por su vertiente positiva para no desanimar a los alumnos

- Evitar un trato distante, eliminar tensiones

- No escribir mensajes demasiado largos

- Resaltar las ideas en los mensajes usando recursos tipográficos o de estilo como bullet points o texto en negrita

- Fomentar un ambiente de comunidad
}

con los alumnos, el profesor o tutor, necesitará disponer de todo el material antes de que empiece el curso para preparar su papel durante el desarrollo del mismo y poder planear la interacción y la comunicación con los alumnos y la interacción de éstos con los materiales. Todo esto significa que hasta el mínimo detalle debería estar calculado al milímetro con antelación al inicio del curso para poder hacer frente a las incidencias que pueden surgir a lo largo de éste.

Por otro lado, y como consecuencia de estas particularidades, desde el punto de vista económico hay que tener en cuenta que la inversión de recursos en la fase de planificación va a ser más alta que en un curso presencial. Como contrapartida, cabe decir que si se pueden realizar varias ediciones sin hacer demasiados cambios -especialmente en los materiales didácticos-, se podrá amortizar esta inversión inicial debido a que el coste marginal de cada nuevo curso puede ser más reducido que en un curso presencial.

\section{Equipos de trabajo}

Así como en actividades de formación presencial se acostumbra a dejar una buena parte del trabajo en manos del profesor -que muchas veces es quien selecciona los contenidos, los redacta, los prepara y los explica-, en la formación on line es indispensable contar con uno o varios equipos de trabajo para preparar y ejecutar activi- 
dades de esta formación. En opinión de Moore [4], se necesitan cuatro equipos para cubrir con éxito cada uno de los cuatro ámbitos de trabajo en un departamento o sistema de formación on line. Evidentemente se puede hacer con menos profesionales -iy se hace!-, pero en instituciones de tamaño medio o grande como puedan ser las universidades, esos cuatro grupos de trabajo o subdepartamentos son realmente necesarios para ofrecer un servicio de calidad.

Estos cuatro equipos de trabajo son:

- Un equipo para diseñar la actividad de formación on line. Puede estar formado por diseñadores, dibujantes, informáticos, expertos en las materias sobre las que trata el curso (como autores de textos), técnicos de formación, pedagogos o los llamados diseñadores instruccionales, un perfil profesional que ha aparecido con el auge de la formación on line y que es experto en diseñar acciones formativas en este tipo de contextos.

- Un segundo equipo para impartir la actividad formado por profesores y tutores. Es el que está en contacto con los alumnos a lo largo del curso. Su misión es guiar y motivar a éstos.

- Otro equipo dedicado a administrar la actividad, formado por técnicos de formación, personal de administración e informáticos. Este equipo gestiona las matrículas, las altas y bajas en el sistema y garantiza un correcto funcionamiento del entorno virtual de aprendizaje.

- Finalmente, un cuarto equipo para coordinar estos otros tres equipos y que está formado por los directores y coordinadores del curso, expertos en formación on line y el/los gestor/es del proyecto.

\section{Funciones del profesor on line}

El profesor que realiza la formación presencial se puede dedicar a la preparación de las clases de manera escalonada, si así lo desea, entre sesión y sesión, aunque esto no sea siempre así. Incluso puede suplir algunas negligencias en la preparación de las clases con un poco de ingenio o con algún cambio de última hora. El profesor es la fuente de información -expone los contenidos, los explica, los ejemplifica, etc.-, y esto le da un margen de maniobra importante. En formación on line, en cambio, este papel lo desempeñan en gran medida los materiales didácticos en sus diferentes formatos, ya sean texto o materiales multimedia. El papel del profesor on line no consiste en exponer los contenidos del curso, tiene más que ver con guiar al alumno a través del entorno y los materiales, facilitar el proceso de aprendizaje y motivar a éste con intervenciones en los momentos precisos.

Este cambio de papel tiene ciertas implicaciones, una de ellas es que hay menos lugar para la improvisación, especialmente sobre los contenidos del curso, los cuales no se podrán modificar de un día para otro, probablemente ni de una semana para otra en función del formato de los materiales. Esto significa que todos aquellos recursos con los que un profesor suple o resuelve las carencias o problemas que pueden surgir a medida que adelanta un curso -errores en el diseño del curso- son de más difícil aplicación en un curso on line.

En esta línea de gestión de las dinámicas docentes, Stephenson et al [5] describen las cuatro funciones clave que debe asumir el profesor on line para el desarrollo correcto de una actividad: pedagógica, social, gestora y tecnológica. Lo que sigue es una simplificación y adaptación del original:

\section{- Pedagógica:}

- Fomentar que el estudiante cree y relacione conocimientos.

- Provocar que el estudiante reflexione.

- Ayudar a identificar puntos y conceptos importantes en el temario.

- Proporcionar feedback.

- Hacer síntesis de los contenidos.

- Social:

- Crear un buen clima en el aula.

- Fomentar sentimiento de comunidad.

- Mostrar un tono positivo.

- Fomentar el sentido del humor en la medida de lo posible.

- Mostrar empatía.

- Gestora:

- Coordinar tareas.

- Gestionar los canales de comunicación.

- Gestionar la secuenciación y temporalización del curso.

- Tecnológica:

- Ayudar al usuario a orientarse dentro de la plataforma de aprendizaje.

- Explicar las limitaciones del sistema. 
Tabla II. Tipos de plataformas de aprendizaje.

\begin{tabular}{|c|c|c|c|}
\hline & Plataforma open source & Plataforma comercial & Plataforma a medida \\
\hline \multirow{2}{*}{ Ventajas } & $\begin{array}{l}\text { Los productos open source } \\
\text { suelen ser gratis o más baratos }\end{array}$ & \multirow{2}{*}{ Implementación más rápida } & Prestaciones y aspecto a medida \\
\hline & $\begin{array}{l}\text { Acceso al código fuente: se puede } \\
\text { adaptar a tus necesidades }\end{array}$ & & Ahorro en licencias de software \\
\hline \multirow{2}{*}{ Desventajas } & $\begin{array}{l}\text { Modificar el código fuente de } \\
\text { un producto creado por otros } \\
\text { puede ser muy laborioso y caro }\end{array}$ & $\begin{array}{l}\text { Coste del producto (caro) } \\
\text { Menos flexibilidad }\end{array}$ & $\begin{array}{l}\text { Alta inversión en diseño e } \\
\text { implementación del producto }\end{array}$ \\
\hline & $\begin{array}{l}\text { Dificultad de adaptar las nuevas } \\
\text { versiones a tu tipo de producto }\end{array}$ & $\begin{array}{l}\text { Dependencia del servicio } \\
\text { técnico del producto }\end{array}$ & $\begin{array}{l}\text { Producto técnicamente } \\
\text { no contrastado }\end{array}$ \\
\hline
\end{tabular}

\section{Plataformas de formación on line}

La plataforma de aprendizaje o entorno virtual de aprendizaje (EVA) es el espacio virtual -lo que en lenguaje técnico se denomina una aplicación weben el cual se desarrolla la actividad de formación. Es un espacio web protegido por contraseña y equipado con los recursos necesarios para el desarrollo de una actividad de formación on line. En este espacio se cuelgan los materiales didácticos y a través de él los participantes se comunican.

En el mercado de las plataformas de aprendizaje existe bastante oferta, los productos que se pueden encontrar se definen a partir de dos parámetros: el acceso al código fuente y el coste de la aplicación. En función del acceso al código fuente, se puede hablar de dos grandes bloques:

- Plataformas open source o de código abierto: el código fuente en estas plataformas -los ficheros que integran el programa- es accesible $y$, por tanto, puede ser modificado. Esto posibilita que uno mismo pueda personalizarlo a su gusto. La más popular es Moodle, seguida por otras como Sakai, Claroline o Atutor.

- Plataformas propietarias: el código fuente en estas plataformas no puede ser modificado por uno mismo, por lo tanto, dependemos del servicio técnico de la compañía proveedora. Blackboard y WebCT son algunas de las más populares.
En función del coste de la aplicación existen dos grupos de plataformas: las gratuitas y las de pago. Normalmente, las plataformas open source son grautitas y las plataformas propietarias son de pago, pero no siempre es así. Fronter, por ejemplo, es una aplicación open source de pago.

Otra opción consiste en hacerse uno mismo (el propio departamento informático) una plataforma a medida que cumpla con las necesidades y requerimientos que se definan a priori. Cada una de estas opciones tiene sus pros y sus contras, que se pueden ver en detalle en la tabla II.

Actualmente se vive un auge de las soluciones open source, una de las cuales, Moodle, se está convirtiendo a una velocidad vertiginosa en el estándar de facto, ya que lo están adoptando organizaciones tan prestigiosas como la Open University inglesa. Parece ser que este fenómeno va en aumento y, cada vez más, las grandes instituciones se inclinan por soluciones open source.

Para obtener más información sobre las plataformas, el sitio web Edutools [6] dispone de datos y comparaciones entre productos. Para saber más sobre EVA open source, véase el informe completo de la consultora británica EPIC [7].

\section{Blended Learning}

El Blended Learning o B-Learning es una combinación de formación presencial y formación on line. 
Tal como lo describen sus valedores, se trata de una estrategia de formación que combina lo mejor de cada método para construir una solución de enseñanza de alta calidad y eficiencia. Básicamente consiste en unir la comodidad y flexibilidad de la formación on line-materiales colgados en Internet, foros, reducción de desplazamientos, etc.- con el alto poder motivador gracias a la interacción física que tiene la formación presencial y que favorece la creación de vínculos entre los participantes.

Un ejemplo de actividad de Blended Learning sería un curso de 50 horas de duración con tres encuentros presenciales a lo largo de tres meses donde los participantes se conocerían y podrían verse y charlar. Estas sesiones presenciales podrían tener como objetivo debatir los conceptos básicos y aplicarlos a casos concretos. Entre sesión y sesión los alumnos podrían trabajar los contenidos del curso a través de los materiales didácticos y de las actividades autoformativas disponibles en el EVA del curso.

\section{Web 2.0 y formación on line, o e-learning 2.0}

Si simplificamos mucho, se puede decir que web 2.0 [8] es la expresión acuñada para referirse a aquellas aplicaciones web, como pueden ser Wikipedia o Blogger, que se centran en el usuario, de modo que éste tiene un papel protagonista en la evolución y modificación de las mismas. Cuando hablamos de web 2.0 nos referimos a este conjunto de aplicaciones o páginas web que tienen un marcado carácter participativo y social, y no sólo a las aplicaciones concretas, sino para referirnos en general a este nuevo modelo basado en comunidades de usuarios.

Esta nueva generación de aplicaciones web ha tenido y está teniendo mucha influencia en el modo en que evoluciona la formación on line, incluso se ha acuñado el término e-learning 2.0 para hacer referencia a la combinación de formación on line y enfoques basados en la web 2.0. En general, cuando se habla de e-learning 2.0 se hace referencia no sólo a la aplicación de tecnologías de la web 2.0 como blogs y wikis para soluciones de formación on line, sino también a un enfoque metodológico de la formación basado en la participación de los alumnos y en actividades y software colaborativo. Para más información sobre el tema, véase el artículo de Downes [9].

\section{Algunas trampas y mitos de la formación on line}

La aparición de la formación on line y la manera como se presentó en sociedad a finales de los noventa, que generó muchas expectativas y con muchas promesas incumplidas, ha dado lugar a muchos malentendidos, algunos de los cuales citamos a continuación.

Uno de los pronósticos más repetidos fue que la formación on line sustituiría a la enseñanza tradicional. Aunque la formación on line está modificando el mercado de la formación, ahora parece bastante evidente que la formación presencial no desaparecerá: hay facetas que la formación on line no puede sustituir satisfactoriamente; más bien tendríamos que ver los dos tipos de formación como complementarios.

Otro mito muy repetido en su momento, aunque últimamente pierde fuerza, es la creencia que la formación on line es más barata que la formación presencial. Esto es falso. Lo que sí es cierto es que un curso on line de mala calidad puede hacerse con un coste menor que un curso presencial de calidad.

Con respecto a las trampas, existen dos enfoques que debemos evitar cuando diseñemos una propuesta de formación on line:

- Situar la tecnología por encima de las personas. Por más sofisticados que sean una plataforma o unos materiales didácticos, no tendremos garantizada la calidad del curso. Lo importante es el diseño del curso, la planificación y el enfoque pedagógico.

- Trasladar a la formación on line ciertos estilos y tecnologías propios de la formación presencial. Por ejemplo, usar diapositivas de PowerPoint para exponer algunos contenidos.

Para conocer una relación de causas que pueden hacer fracasar una propuesta on line, véase el interesante artículo de Woodill [10].

\section{Conclusión}

La formación on line es actualmente la modalidad predominante de educación a distancia. Aunque no sustituirá a la formación presencial, la tendencia es que cualquier actividad formativa de calidad integre componentes on line o tecno- 
lógicos. Esta formación cuenta con un público específico, como son grupos de población distantes geográficamente de los centros educativos, con horarios o situaciones no compatibles con la formación presencial o personas discapacitadas físicamente y con problemas para desplazarse.

El sector de la formación, después de una fase de boom mediático y otra de decepción, se encuentra en un momento favorable, de crecimiento. La fase de decepción ha servido para redimensionar las posibilidades de la formación on line después de un boom tecnológico que creó unas expectativas demasiado altas, expectativas que han pesado como una losa en el despegue del sector. Actualmente, las instituciones educativas y las corporaciones han depositado muchas esperanzas en la formación on line.

\section{Bibliografía}

1. Revuelta M, Esposoz F. La formación tiende al e-learning. Correo Farmacéutico 2008. URL: http://www.correofarmaceutico.com/edicion/correo_farmaceutico/farmaciaeindustria/es/desarrollo/1142372.html [02.09.2007].
2. e-learning Workshops. Según IDC, el e-learning experimentará un crecimiento exponencial hasta el año 2008 URL: http://www.elearningworkshops.com/modules.ph $p ?$ name $=$ News\&file $=$ article\&sid $=613$ [02.09.2007].

3. Cabero J. Bases pedagógicas del e-learning. Revista de Universidad y Sociedad del Conocimiento (RUSC) 2006; vol. 3. UOC. URL: http://www.uoc.edu/rusc/3/1/dt/esp/ cabero.pdf [02.09.2007].

4. Moore MG. Teamwork. The American Journal of Distance Education 2007; 21: 113-6.

5. Stephenson J, Sangrà A. Modelos pedagógicos y e-learning. Barcelona: FUOC.

6. Edutools. Archive CMS: product list. URL: http://www. edutools.info/item_list.jsp?pj=8 [02.09.2007].

7. Aberdour M. Open source learning management systems. Brighton: EPIC; 2007. URL: http://www.epic.co.uk/ content/news/oct_07/whitepaper.pdf [02.09.2007].

8. O'Reilly T. What is web 2.0:designpatterns and business models for the next generation of software. URL: http:// www.oreillynet.com/pub/a/oreilly/tim/news/2005/09/30/ what-is-web-20.html [02.09.2007].

9. Downes S. e-learning 2.0. URL: http://elearn-mag.org/ subpage.cfm?section $=$ articles\&article $=29-1$ [02.09.2007].

10. Woodill G. Where is the learning in e-learning? URL: http://www.e-learningguru.com/wpapers/e-Learning _ analysis.pdf [02.09.2007]. 